\title{
Metaheuristic Approach Based on Neighbourhood Search for Solving P-Median Problem
}

\author{
Srinivas Reddy.D ${ }^{1}$, A. Govardhan ${ }^{2}$ SSVN Sarma ${ }^{3}$ \\ ${ }^{I}$ Associate Professor, Vaageswari College of Engineering, Karimnagar,A.P,India \\ ${ }^{2}$ Professor, Department of CSE, University College of Engineering, JNTU Hyderabad, A.P, India \\ ${ }^{3}$ Professor, Department of CSE, Vaagdevi College of Engineering, Warangal, A.P, India
}

\begin{abstract}
Now a day there is much more amplified curiosity in combinatorial optimization. The p-median problem which is a facility location problem, deals with discrete data and hence it is characterized as a combinatorial optimization problem. It is NP-Hard in nature that ascertains the specified number of locations as facilitators which serves the maximum locations. The p-median problem will be productive in several applications areas such as mounting marketing strategies in Management Sciences and recognition of server positions in computer networks. A new Metaheuristic approach with Neighbourhood Search (NS) technique has been proposed in the present paper which unveil all possible combinations with the elements in the neighbourhood of individual elements in the solution and recognizes the optimal solution i.e., which serves the maximum locations so that the sum of the total distance from the each element to the facilities is minimized. The proposed metaheuristic approach is an iterative one which contains two phases. Construction phase is the first phase that structure the initial solution and based on the initial solution the second phase explore for the optimal solution based on NS approach and then the probable solution space is computed to obtain the optimal solution.
\end{abstract}

Keywords: Metaheuristic, neighbourhood, local search, neighbourhood search, hybridization.

\section{Introduction}

The p-median problem can be depicted as: Let $\mathrm{F}$ is the set of facilities, $\mathrm{C}$ is the set of customers and distance function $d$ is such that $d: C \times F \rightarrow R$. Here the distance function estimates the distance between a customer and a facility. The p-median problem determines a subset $R$ of facilities $F$ such that $|R|=p$, for any positive integer $p$ and number of facilities $n$, where $p \leq n$, such that the sum of the distances from each customer to its adjacent facility is minimized. In the proposed work it is assumed that every customer location can be considered as a facility i.e. $\mathrm{F}=\mathrm{C}$, and also for giving equal importance to each location it is considered that $\mathrm{w}_{\mathrm{i}}=$ 1.

Mathematically p-median problem is stated as [24]

$$
\begin{aligned}
& \text { Minimize } f(d, x)=\sum_{i=1}^{n} \sum_{j=1}^{n} w_{i} d_{i j} x_{i j} \\
& \text { subject to } \sum_{j=1}^{n} x_{i j}=1 \quad \forall i \\
& x_{i j} \leq y_{j} \quad \forall i_{j} j \\
& \sum_{j=1}^{n} y_{j}=p \\
& x_{i j}=0 \text { or } 1 \quad \forall i_{j} j \\
& y_{i}=0 \text { or } 1 \quad \forall j
\end{aligned}
$$

Where

$\mathrm{n}=$ number of locations

$\mathrm{x}_{\mathrm{ij}}=1$ if a location $\mathrm{i}$ is assigned to facility located at $\mathrm{j}$,

$=0$ other wise 
$\mathrm{y}_{\mathrm{i}}=1$ if jth location is a facility

$=0$ other wise

$\mathrm{d}_{\mathrm{ij}}=$ distance measured from location $\mathrm{i}$ to location $\mathrm{j}$

$\mathrm{p}=$ preferred number of locations as facilities

Here the first objective function minimizes the sum total of the distances between the customer locations and the chosen number of locations. The second constraint substantiates that each location is allotted to precisely one adjoining facility. The third constraint forbids the allotment of customer locations to a facility that was not preferred as a desired location. The fourth constraint depict the total number of desired locations as $\mathrm{p}$ and finally the fifth and sixth constraints guarantee that $\mathrm{x}$ and $\mathrm{y}$ are binary valued. As the result of the $\mathrm{p}$ median problem segregate the solution space, the given space can be classified as groups and hence the pmedian problem can be used as a clustering technique.

To discover the solution space of a certain optimization problem effectively several general-purpose sophisticated procedures can be instantiated. Formerly, Metaheuristics like genetic algorithms; tabu search, simulated annealing, ant systems, GRASP and others have been imparted and are applied to real-life problems in numerous areas of science [13]. To elucidate the GRASP (Greedy Randomized Adaptive Search Procedures) Metaheuristic [2,3] several optimization problems [4] are effectively employed. The search procedure for recognizing the solution utilized by GRASP is iterative and each iteration consists of two phases: construction and enhancement phase. The construction phase aims to build a feasible solution, and based on the feasible solution its neighborhood is discovered by the enhancement phase to find a better solution [14][25][26]. The outcome is the principal solution originated over all iterations.

The paper is structured as follows: In section II, the proposed Neighbourhood Search based metaheuristic approach and its phases - Construction and Neighbourhood phases are described in detail. In section III, experimental results and comparisons of optimal values (quality) and execution times are anticipated. Section IV provides the conclusions.

\section{The Grasp Metaheuristic}

GRASP [14] has been adopted by many optimization problems and acquired productive results [4]. GRASP entailed of two- phases which are operated repeatedly. The first phase of GRASP is the construction phase in which an absolute solution is constructed. As this absolute solution is not guaranteed to be locally optimal, in the second phase, a novel enhancement phase is employed. This course is repeated until an annihilation measure is attained and the superlative solution originated over all iterations is taken as final outcome.

The NSApproach logic is illustrated in Figure 1. Initially, the variable to hold the optimal solution instigated is initialized with null set. Then the construction phase is implemented which is adopted from [25] and then the neighborhood search approach is employed to the constructed solution. The quality of the so obtained solution is compared to the current optimal solution found and, if necessary, the optimal solution is updated. Eventually the best optimal solution is returned.

A most modern approach in metaheuristic research is the exploration of hybrid Metaheuristic [21]. One such hybrid scheme results from the assortment of hypothesis and policies from two or more metaheuristics and another one counterparts to Metaheuristics united with concepts and procedures from other areas accountable for performing exclusive tasks that can progress the original method. The hybridization of GRASP with neighbourhood process initially anticipated, introduced and adopted to the set packing problem $[15,16,25]$.

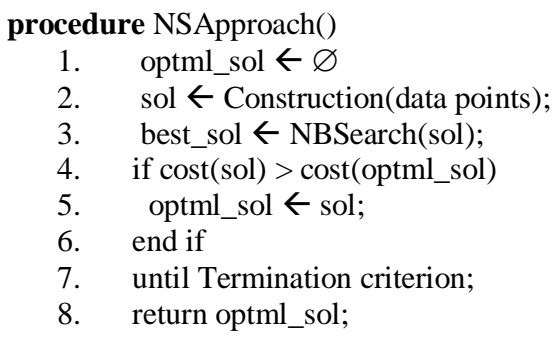

FIGURE 1: NSApproach procedure 


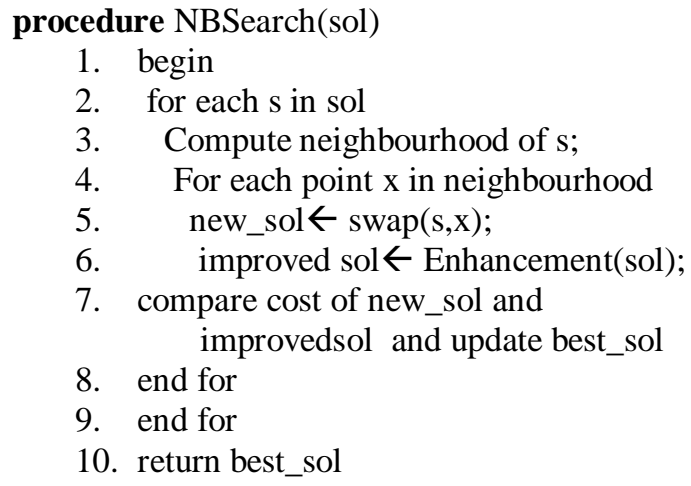

FIGURE 2: NBSearch algorithm



FIGURE 3: Proposed Enhancement Phase used in NBSearch

The logic behind NBSearch phase is described in Figure 2. Here neighborhood of each solution element is determined with radius of 5 units of Euclidian distance from that element. After that the solution element i.e. the neighborhood centre is interchanged with the one of the member in the neighbourhood and remaining elements are kept as it is. Likewise new solution can be achieved. Each and every possible solution is taken and is verified and if necessary, improved by Enhancement phase. This process is repeated for each and every element in the solution. By this it can be specified that the proposed algorithm verifies all possible combinations to get the best optimal solution.

The basic logic of the Enhancement is presented in Figure 3. Initially control variables are assigned with initial values. The cost_eval () function assess the overheads of a solution by identifying the aggregation of the distances amid all customers and their adjoining facilities. Subsequently the neighborhood of the existing solution is organized and if any superior one is found, it transpires into the current one. The same process reiterate again, till no supplementary enhancement is made. It is repeated p times and the best solution found so far is returned. In each iteration, solitary element $R I$ of the solution is interchanged by all elements in the neighborhood to it in its partition $P_{i}$, which contains other than solution elements in the data set. At the present it is assumed that an element $e$ is nearby $R I$ in its partition $P_{i}$ if the distance among $e$ and $R I$ is smallest or equal to the average of distances between $R I$ and all elements in $P i$.

To condense the computational exertion of the enhancement phase, the pronouncement obtained in each swap over is approximately evaluated and the best solution is exactly evaluated. The function t_cost_eval() estimates the outlay of a solution approximately by recalculating the distances within the partition $P_{i}$ only, without making this calculation inside the other partitions. As there is a change of location exact reckoning may be necessary. Then it is assessed for better solution than the current one. If there exists a better solution than the recent solution, the new one transpires to be the current solution and again the enhancement phase incepted.

\section{Experimental Results}

The experimental results acquired for GRASP and NS approach are presented in this section, and the results are compared on the bases of solution quality against p. Experiments are conducted on data sets with 15, 25, 50, 75 points. Results are tabulated and graphs are plotted. The data sets under study are taken from the web site of Professor Eric Taillarrd, Kent University of Applied Sciences of Western Switzerland. The companion website for p-medin problem instances is http://mistic.heigvd.ch/taillard/problemes.dir/location.html. 
In Graph-1 Objective function value i.e. cost for $\mathrm{p}$-median problem is compared using both algorithms GRASP and NS approach for the data set of size 25 with number of facility locations (p) incremented by 3. It is observed that NS approach is working better than GRASP.



In Graph-2 Objective function value i.e. cost for $\mathrm{p}$-median problem is compared using both algorithms GRASP and NS approach for the data set of size 50 with number of facility locations (p) incremented by 10. It is observed that NS approach is working superior than GRASP.

In Graph-3 execution time is compared for both algorithms GRASP and NS approach for the data set of size 15 with number of facility locations (p) incremented by 3 . It is identified that NS approach is taking more processing time than GRASP.

In Graph-4 execution time is compared for both algorithms GRASP and NS approach for the data set of size 25 with number of facility locations (p) incremented by 3 . It is recognized that NS approach is taking more processing time than GRASP.
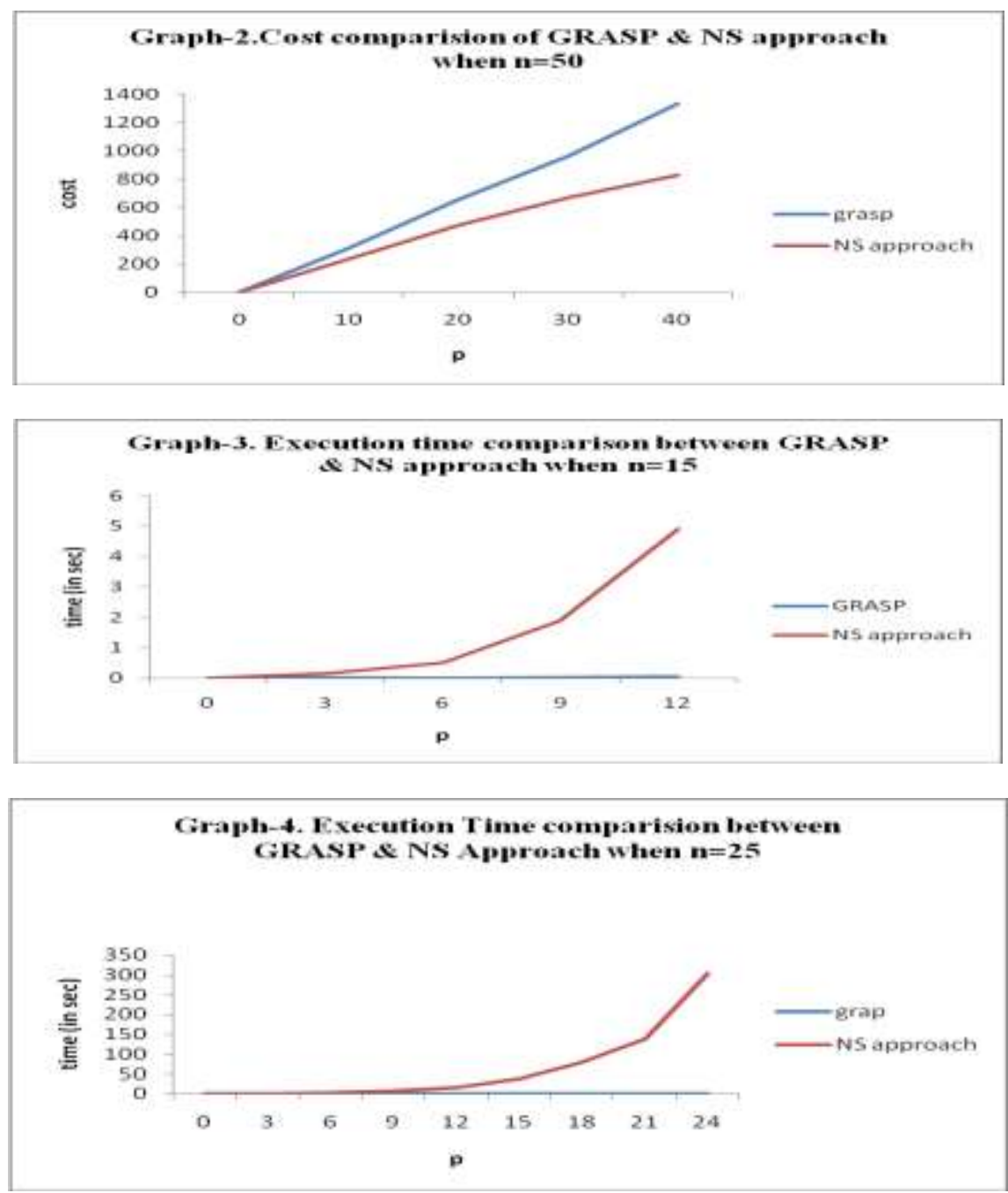


\section{Conclusions}

It is observed that NS approach outperforms GRASP in quality aspect as the data size increases. It is also identified through the experimental results that NS approach consuming more processor time than that of GRASP even though it is good in cost aspect. The main reason behind the more processor time consumption for NS approach is that it is inspecting for all possible combinations within the neighbourhood of individual solution elements but GRASP checks the probable combinations in the solution attained. It is also observed that NS approach outperforms GRASP as the size of the data set increases.

\section{References}

[1] R. Agrwal and R. Srikanth, Fast algorithms for mining association rules, Proceedings of the Very Large Data Bases Conference, pp. 487-499, 1994

T. A. Feo and M. G. C. Resende, A probabilistic heuristic for a computationally difficult set covering problem, Operational Reseach Letters, 8 (1989), pp.67-71.

[3] T. A. Feo and M. G. C. Resende, Greedy randomized adaptive search procedures, Journal of Global Optimization, 6 (1995), pp. 1609-1624.

[4] T. A. Feo and M. G. C. Resende, GRASP: An annotated bibliography, Essays and Surveys in Metaheuristics, Kluwer Acadamic Publishers, 2002

[5] M. D. H. Gamal and Salhi, A cellular heuristic for the multisource Weber Prolem, computers \& Operations Research, 30 (2003), pp.1609-1624

[6] B. Geothals and M. J. Zaki, Advances in Frequent Item set Mining Implementations: Introduction to FIMI03, Proceedings of the IEEEICDM workshop on Frequent Item set Mining Implementations, 2003.

[7] G. Grahne and J. Zhu, Efficiently using prefix-trees in mining frequent item-sets, Proceedings of the IEEEICDM Workshop on Frequent Itemset Mining Implementations, 2003

[8] J. Han, J. Pei and Y. Yin, Mining frequent patterns without candidate generation, Proceedings of the ACMSIGMOD International conference on Management of Data, pp. 1-12, 2000.

[9] J. Han and M. Kamber, Data Mining: Concepts and Techniques, $2^{\text {nd }}$ Ed., Morgan Kaufman Publishers, 2006

[10] O. Kariv and L. Hakimi, An algorithmic approach to network location problems, part ii: the p-medians, SIAM Journal of Applied Mathematics, 37 (1979), pp.539-560

[11] N. Mladenovic, J. Brimberg, P. Hansen and Jose A. Moreno-Perez, The p-median problem: A survey of metaheuristic approaches, European Journal of Operational Research, 179 (2007), pp.927-939.

[12] S. Orlando, P. Palmerimi and R. Perego, Adaptive and resource-aware mining of frequent sets, Proceedings of the IEEE International conference on Data Mining, pp.338-345, 2002

[13] I. Osman and G. Laporte, Metaheuristics: A bibliography, Annals of Operations Research, 63 (1996), pp. $513-623$.

[14] M. G. C. Resende and C. C. Ribeiro, Greedy randomized adaptive search procedures, Handbook of Metaheuristics , Kulwer Academic Publishers, 2003.

[15] M. H. F. Ribeiro, V. F. Trindade, A. Plastino and S. L. Martins, Hybradization of GRASP metaheuristic with datamining techniquess, Proceedings of the ECAI Workshop on Hybrid Metaheuristics, pp.69-78,2004.

[16] M. H. F. Ribeiro, V. F. Trindade, A. Plastino and S. L. Martins, Hybrazation of GRASP Metaheuristic with data mining techniques, Journal of Mathematical Modelling and Algorithms, 5 (2006), pp.23-41.

[17] S. Salhi, Heuristic Search: the Science of Tomorrow, OR48 Keynote Papers, Operational Research Society, pp.38-58, 2006.

[18] L. F. Santos, M. H.F. Ribeiro, A. Plastinoand S. L. Martins, A hybrid GRASP with data mining for the maximum diversity problem, proceedings of the International Workshop on Hybrid Metaheuristics, LNCS 3636, pp. 116-127, 2005.

[19] L.F. Santos, C.V.Albuquraque, s. L. Martins and A. Plastino, A hybrid GRASP with data mining for efficient server replication for reliable multicast, Proceedings of the IEE GLOBECOM Conference, 2006.

[20] L. F. Santos, S. L. Martins and A. Plastino, Applications of the DM-GRASP heuristic: A survey, International Transanctions in Operational Research, 15 (2008), p.387-416.

[21] E. G. Talbi, A taxonomy of hybrid metaheuristics, Journal of Heuristics, 8 (2002), pp.541-564

[22] B. C. Tansel, R. L. Fransis, and T. J. Lowe. Location on networks: A survey, Management Science, 29 (1983),pp.482-511.

[23] I. H.Witten and E.frank, DataMining: Practical Machine Learning Tools nd Techniques. $2^{\text {nd }}$ Ed., Morgan Kaufmann Publishers, 2005

[24] Moh'd Belal Al-Zoubi, Ahmed Sharieh, Nedal Al-Hanbali and Ali Al-Dahoud, a Hybrid Heuristic Algorithm for Solving the PMedian Problem. Journal of Computer Science (Special Issue) 80-83, 2005, Science Publications.

[25] Alexndre Plastino, Eric R Fonseca, Richard Fuchshuber, Simone de L Martins, Alex.A.Freitas, Martino Luis and Said Salhi A Hybrid Datamining Metaheuristic for the p-median problem. Proceedings of SIAM journal (Data Mining), 2009.

[26] Sunil Nadella, Kiranmai M V S V, Dr Narsimha Gugulotu, A Hybrid K-Mean-Grasp For Partition Based Clustering Of TwoDimensional Data Space As An Application of P-Median Problem, International Journal of Computer and Electronics Research [Volume 1, Issue 1, June 2012] ISSN : 2278-5795 